\title{
Interplanetary Protons versus Interacting Protons in the 2017 September 10 Solar Eruptive Event
}

\author{
Leon Kocharov ${ }^{1}$ (10), Melissa Pesce-Rollins ${ }^{2}$ (10), Timo Laitinen ${ }^{3}$ (), Alexander Mishev ${ }^{1,4}$, Patrick Kühl ${ }^{5}$, Andreas Klassen ${ }^{5}$, \\ Meng Jin ${ }^{6,7}$ (i), Nicola Omodei ${ }^{8}$ (iD), Francesco Longo ${ }^{9,10}$ (i), David F. Webb ${ }^{11}$ (i), Hilary V. Cane ${ }^{12}$, Bernd Heber ${ }^{5}$ (i), \\ Rami Vainio $^{13}$, and Ilya Usoskin ${ }^{1,4}$ (B) \\ ${ }^{1}$ Sodankylä Geophysical Observatory, University of Oulu, P.O.B. 3000, Oulu FI-90014, Finland \\ ${ }^{2}$ Istituto Nazionale di Fisica Nucleare, Sezione di Pisa, I-56127 Pisa, Italy \\ ${ }^{3}$ Jeremiah Horrocks Institute, University of Central Lancashire, Preston PR1 2HE, UK \\ ${ }^{4}$ Space Climate Research Unit, University of Oulu, Oulu FI-90014, Finland \\ ${ }^{5}$ Institut für Experimentelle und Angewandte Physik, Christian-Albrechts-Universität, Kiel D-24118, Germany \\ ${ }^{6}$ Lockheed Martin Solar and Astrophysics Lab, Palo Alto, CA 94304, USA \\ ${ }^{7}$ SETI Institute, Mountain View, CA 94043, USA \\ ${ }^{8}$ W. W. Hansen Experimental Physics Laboratory, Kavli Institute for Particle Astrophysics and Cosmology, Department of Physics and SLAC National Accelerator \\ Laboratory, Stanford University, Stanford, CA 94305, USA \\ ${ }^{9}$ Istituto Nazionale di Fisica Nucleare, Sezione di Trieste, I-34127 Trieste, Italy \\ ${ }^{10}$ Dipartimento di Fisica, Università di Trieste, I-34127 Trieste, Italy \\ ${ }^{11}$ ISR, Boston College, Chestnut Hill, MA 02459, USA \\ ${ }^{12}$ Department of Mathematics and Physics, University of Tasmania, Hobart, Tasmania, Australia \\ ${ }^{13}$ Space Research Laboratory, Department of Physics and Astronomy, University of Turku, Turku FI-20014, Finland \\ Received 2019 May 23; revised 2020 January 4; accepted 2020 January 5; published 2020 February 7
}

\begin{abstract}
We analyze the relativistic proton emission from the Sun during the eruptive event on 2017 September 10, which caused a ground-level enhancement (GLE 72) registered by the worldwide network of neutron monitors. Using the neutron monitor data and interplanetary transport modeling both along and across interplanetary magnetic field (IMF) lines, we deduce parameters of the proton injection into the interplanetary medium. The inferred injection profile of the interplanetary protons is compared with the profile of the $>100 \mathrm{MeV} \gamma$-ray emission observed by the Fermi Large Area Telescope, attributed to pion production from the interaction of $>300 \mathrm{MeV}$ protons at the Sun. GLE 72 started with a prompt component that arrived along the IMF lines. This was followed by a more prolonged enhancement caused by protons arriving at the Earth across the IMF lines from the southwest. The interplanetary proton event is modeled using two sources-one source at the root of the Earth-connected IMF line and another source situated near the solar western limb. The maximum phase of the second injection of interplanetary protons coincides with the maximum phase of the prolonged $>100 \mathrm{MeV} \gamma$-ray emission that originated from a small area at the solar western limb, below the current sheet trailing the associated coronal mass ejection (CME). A possible common source of interacting protons and interplanetary protons is discussed in terms of proton acceleration at the CME bow shock versus coronal (re-)acceleration in the wake of the CME.
\end{abstract}

Unified Astronomy Thesaurus concepts: Solar coronal mass ejections (310); Solar energetic particles (1491); Solar particle emission (1517); Solar coronal mass ejection shocks (1997); Solar flares (1496); Solar gamma-ray emission (1497)

\section{Introduction}

Solar flares and coronal mass ejections (CMEs) can accelerate ions up to relativistic energies, as evidenced by observations of solar $\gamma$-rays and neutrons produced by highenergy ions colliding in the solar atmosphere and from observations of accelerated ions arriving at 1 au. However, a relationship between the solar interacting particles and the solar energetic particles (SEPs) in space is a matter of controversy (e.g., Lin 2005). The ratio of the number of ions escaping into the interplanetary medium $\left(N_{\uparrow}\right)$ to the number precipitating into the solar chromosphere $\left(N_{\downarrow}\right)$ may vary from one flare to another by a few orders of magnitude, with larger $N_{\uparrow} / N_{\downarrow}$ values associated with gradual (eruptive) flares compared to impulsive ones (Murphy \& Ramaty 1984; Hua \& Lingenfelter 1987; Ramaty et al. 1993).

In extreme SEP events, protons with energies $>400 \mathrm{MeV}$ are produced. These high-energy particles can trigger a nuclear cascade in the atmosphere of the Earth and thus be detected by ground-based detectors like neutron monitors. These are known as ground-level enhancements (GLEs; e.g., Shea \& Smart 2012; Poluianov et al. 2017, and references therein). Protons of nearly the same energies can trigger nuclear interactions at the Sun, and their neutral secondaries, like pion-decay $\gamma$-rays, can be detected on board a spacecraft. The Fermi Large Area Telescope (LAT; Atwood et al. 2009) has observed a number of long-duration solar $\gamma$-ray flares, including multi-hour-long flares (Ackermann et al. 2014, 2017; Ajello et al. 2014). One such event was the flare on 2017 September 10 (Omodei et al. 2018), which was also associated with a GLE.

The 2017 September 10 GLE (GLE 72) has been analyzed by a number of researchers including Mishev et al. (2018) and Jiggens et al. (2019). This eruptive flare was very well observed and its different facets are now described in many publications. In particular, detailed, broadband studies of the CME genesis, magnetic morphology, and dynamics (Gopalswamy et al. 2018; Long et al. 2018; Veronig et al. 2018), high-resolution imagery of the global EUV wave (Liu et al. 2018), and spectroscopic observations of the current sheet in the wake of the eruption (Cheng et al. 2018; Li et al. 2018; 

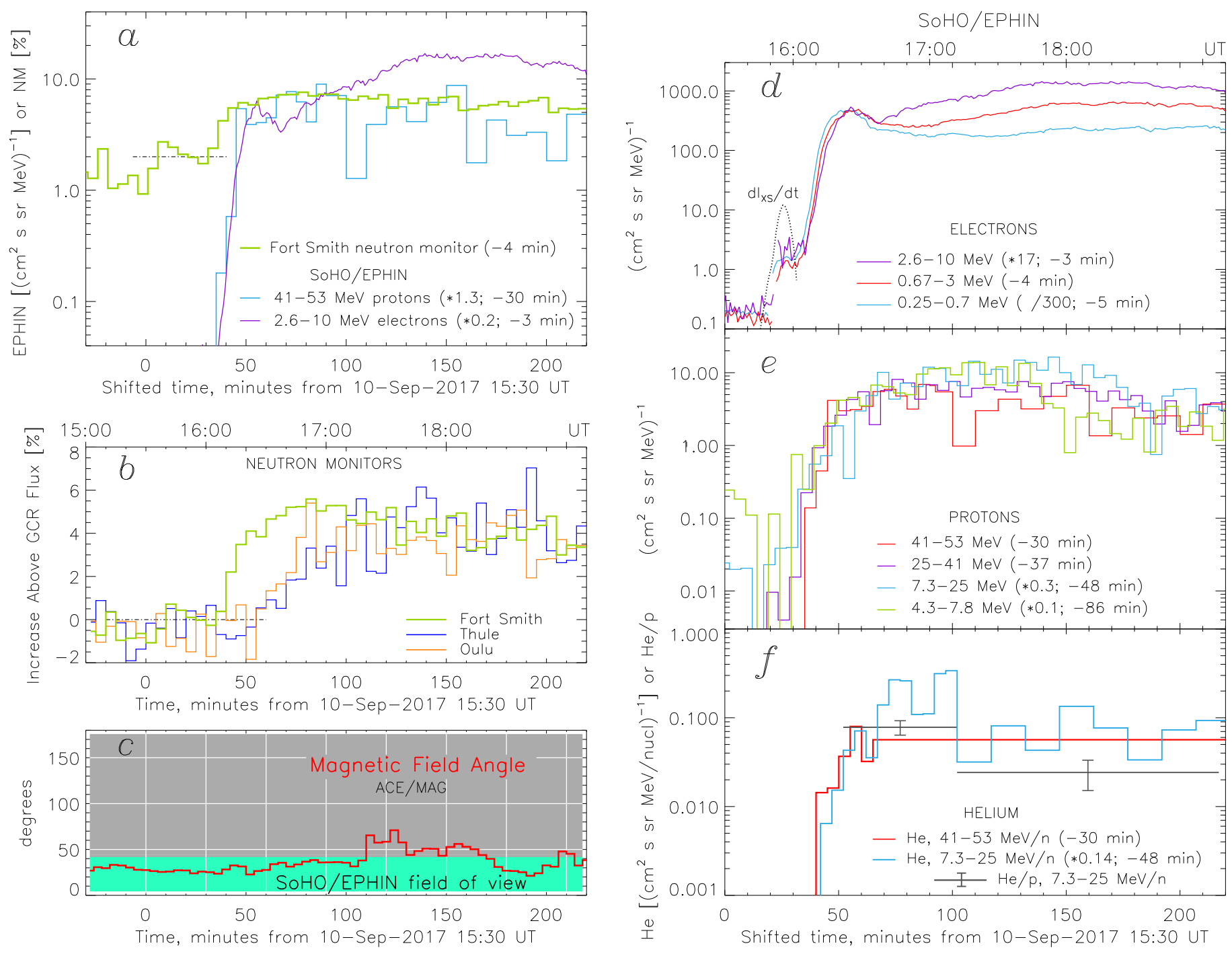

Figure 1. Overview of the SEP event. (a), (d)-(f) Time-shifted and renormalized profiles of different particle species. A time shift is the ion/electron travel time of 1.37 au minus the Sun-Earth flight time of photons. In panel (a), 98\% of the galactic cosmic ray (GCR) contribution to the neutron monitor count rate is subtracted (the dotted-dashed line indicates the remnant average background). In panel (f), additionally shown is the helium-to-proton abundance ratio. (b) Non-shifted time profiles of three polar neutron monitors with $100 \%$ of the GCR contribution subtracted. (c) Angle between the axis of the SoHO/EPHIN view cone and the IMF direction measured on the Advanced Composition Explorer (the level 2 ACE/MAG data at http://www.srl.caltech.edu). The green sector shows the EPHIN view cone for the SEP registration. In panel (d), the flare's impulsive phase is illustrated with the time derivative of the soft X-ray emission (0.05-0.4 nm; GOES 13). During the impulsive phase, the electron channels of SoHO/EPHIN are contaminated by the hard X-ray emission.

Seaton \& Darnel 2018; Warren et al. 2018; Yan et al. 2018) are available. Our present study focuses on high-energy protons at the Sun and in the interplanetary medium in conjunction with flare-CME imagery and spectroscopic observations.

\section{High-energy Particle and Gamma-Ray Data}

A major SEP event was registered by particle instruments in space and neutron monitors (NMs) on the ground up to proton energies $\approx 4 \mathrm{GeV}$. We use the world NM network data available in the Oulu database (http://gle.oulu.fi) and data from the Electron Proton Helium Instrument (EPHIN) on the Solar and Heliospheric Observatory $(\mathrm{SoHO})$ spacecraft that is a stabilized platform outside the Earth's magnetosphere (Müller-Mellin et al. 1995; Kühl \& Heber 2019).

An overview of the SEP-GLE event is shown in panel (a) of Figure 1. The GLE rose steeply during 16:10-16:20 UT. However, the early part of the GLE was reliably detected by only one neutron monitor, Fort Smith (panel (b)). A statistically significant signal from a larger number of NMs was observed after 16:25 UT, allowing derivation of the spectral and angular characteristics of solar protons with good accuracy. Panels (d)-(f) respectively are for the electrons, protons, and helium intensities measured by EPHIN. At the start of the event, the EPHIN field of view was along the interplanetary magnetic field (IMF) line (panel (c)). Thus, EPHIN would detect the first particles arriving at $1 \mathrm{au}$. The proton panel (e) represents a kind of velocity dispersion analysis (VDA) of the event's rise phase, that is, the observed intensities of different energy channels are plotted with the particle registration time shifted back for the Sun-Earth travel time with the traveled distance adjusted so that the count-rate profiles of all energy channels increase simultaneously. Then, the Sun-Earth flight time of photons is added to the shifted time in order to compare the SEP profiles with observed solar electromagnetic emissions. Correspondingly shifted profiles of high-energy protons, electrons, and helium are additionally shown in panels (a), (d), and (f). In the 
latter panel, we also show the helium-to-proton abundance ratio observed by EPHIN.

Modeling of the SEP transport from the solar source to the Earth's orbit shows that at large values of the particle mean-free path, $\Lambda \gtrsim 3 \mathrm{au}$, the rise profile of the particle source may be well estimated by a simple shifting of the near-Earth SEP profile back in time for the particle travel time from Sun to Earth (in contrast to the case of $\Lambda \lesssim 1$ au in which the interplanetary scattering blurs off the "image" of the solar source and a regular deconvolution procedure is required; Kocharov et al. 2015a). The time-shift value and the corresponding average distance traveled by SEPs depend on $\Lambda$. In the case of the 2017 September 10 event, the VDAestimated traveled distance is 1.37 au (Figure 1(e)), which, with the interplanetary transport modeling and particle sampling into the EPHIN's view cone (Figure 1(c)), implies the mean-free path $\Lambda=3$ au.

The SEP event begins with simultaneous injection of protons and helium in a wide energy range, from a few $\mathrm{MeV}$ to a few $\mathrm{GeV}$, and emission of relativistic electrons, which start to rise next to the end of the flare's impulsive phase (Figure 1). The electron profiles shown in panel (d) reveal also a second component of a harder energy spectrum that appears $\sim 30$ minutes after the start of the first injection. We also note that the SEP event starts with a helium-rich composition: $\mathrm{He} / \mathrm{p}$ $\approx 0.08$ (panel (f)).

Figure 2 shows the results of the $>100 \mathrm{MeV} \gamma$-ray detection by Fermi-LAT (Omodei et al. 2018). The light curve in the upper panel comprises: (1) the flare pulse, (2) the postimpulsive, gradual rise $2 \mathrm{a}$ ending with the partly observed steep rise $2 b$, and (3) the extended, multi-hour long $\gamma$-ray burst observed in several successive orbits of Fermi. The partly observed rise $2 \mathrm{~b}$ most likely is the leading front of a post-flare $\gamma$-ray pulse that is a counterpart of the microwave burst observed at 16:26-16:50 UT by the Expanded Owens Valley Solar Array (EOVSA; Gary et al. 2018, Figure 1 therein; mw2 in our figure).

We use the LAT observations of the pion-decay emission to estimate the corresponding numbers of the pion-producing protons. The pion-decay templates used in the LAT-data fits depend on the ambient density, composition, and magnetic field, and on the accelerated particle composition, pitch-angle distribution, and energy spectrum. The templates represent a particle population with an isotropic pitch-angle distribution and a power-law energy spectrum, interacting in a thick target with a coronal composition (Reames 1995a), taking the helium abundance ratio $\mathrm{He} / \mathrm{H}=0.1$. The templates we use are based on a detailed study of the $\gamma$-rays produced from pion decay (updated from Murphy et al. 1987). The results are shown in the two lower panels of Figure 2. In what follows, we will focus on the delayed, very prolonged emission of Phase 3 . The energy spectrum of the interacting protons of Phase 3 is initially harder than that in Phase 2 (lower panel). This may be explained by a new acceleration or reacceleration during the data-gap period preceding the first observed interval of Phase 3. That acceleration was operating either simultaneously with the microwave burst mw2 or shortly after it. Note between the first two data points of Phase 3 that there is a concurrent softening of the interacting proton spectrum and an increase of the interacting proton number. This may be due to an enhancement of the proton precipitation rate into the chromosphere with no further acceleration.

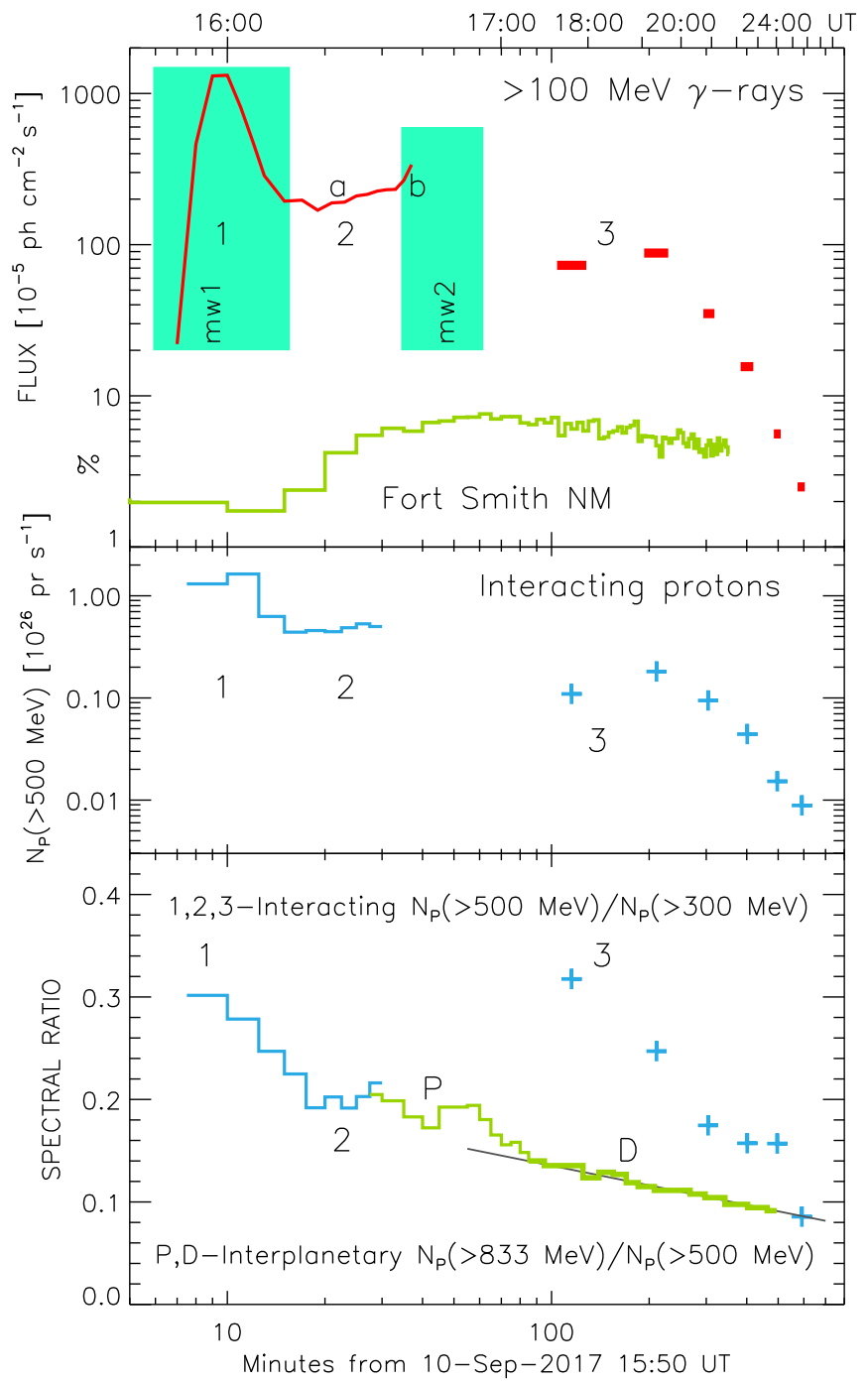

Figure 2. Upper panel: the $>100 \mathrm{MeV} \gamma$-ray flux of the 2017 September 10 flare with data of Fermi/LAT and the GLE profile observed by the Fort Smith neutron monitor (in percent of the GCR contribution, with $98 \%$ of that contribution subtracted). The timing of two major microwave bursts is additionally indicated with green strips: the flare pulse, mw1, shown for $16.45 \mathrm{GHz}$ and the delayed burst, mw2, shown for $5.38 \mathrm{GHz}$ (Gary et al. 2018). Middle panel: the inferred number of protons interacting at the Sun to produce $\gamma$-ray emissions of Phases 1,2, and 3. Lower panel: the proton spectral ratio $N_{\mathrm{p}}(>500 \mathrm{MeV}) / N_{\mathrm{p}}(>300 \mathrm{MeV})$ as estimated with Fermi/LATdata for the interacting protons and $N_{\mathrm{p}}(>833 \mathrm{MeV}) / N_{\mathrm{p}}(>500 \mathrm{MeV})$ estimated with the NM network data for the interplanetary protons. The interplanetary spectrum is taken at somewhat higher energies compared to the interacting spectrum, because NMs are not sensitive to the proton flux below $400 \mathrm{MeV}$. The accuracy of the spectral ratio determination is $5 \%-10 \%$. The GLE's prompt component and delayed component are respectively labeled with $\mathrm{P}$ (thin line) and $\mathrm{D}$ (thick line).

Processing of the neutron monitor network data is done with an empirical fitting of the observed count-rate profiles, with templates depending on the solar proton spectrum, the proton flux axis direction, and the pitch-angle distribution outside the Earth's magnetosphere. The differential intensity of solar protons is fitted with a modified power-law function of the proton rigidity: $J(P) \sim P^{-(\gamma+\delta \gamma(P-1))}$, where rigidity $P$ is measured in $\mathrm{GV} ; \delta \gamma>0$ can describe possible steepening of the spectrum with increase of rigidity. The proton pitch-angle distribution is fitted with the sum of two Gaussian functions-a Gaussian function of width $\sigma_{1}$ describes the particle streaming 

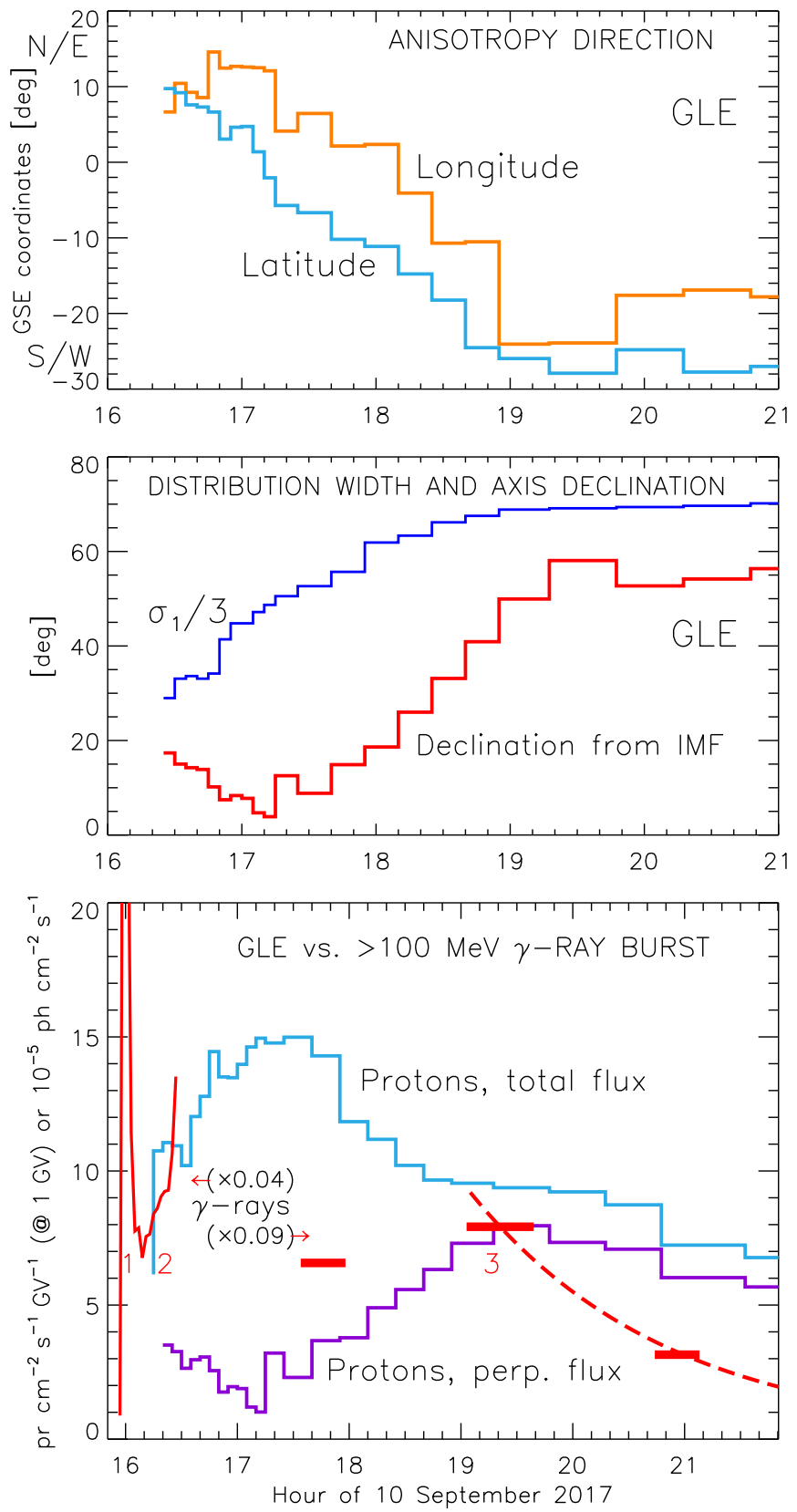

Figure 3. Time profiles of the interplanetary proton flux and the solar $\gamma$-ray emission. Upper panel: direction of the interplanetary proton flux in the GSE coordinates. Middle panel: width of the proton pitch-angle distribution, $\sigma_{1}$ (Mishev et al. 2018), and the angle between the proton flux and the interplanetary magnetic field ( $A C E /$ MAG gliding 100 minute average data). Lower panel: total flux of the interplanetary protons and its component perpendicular to the magnetic field as compared with the $\gamma$-emission profile (red curve and bars). The decl. angle error of the proton flux is about $15^{\circ}$, so a perpendicular flux less than $0.3 \times$ the total flux cannot be resolved. Observable perpendicular flux appeared from 18:00 UT. The red dashed line fits the exponential decay of the $\gamma$-ray flux continuing for many hours after 19:00 UT (Figure 1 by Omodei et al. 2018).

from the Sun along the symmetry axis, not necessarily parallel to the IMF line, and the second Gaussian can account for possible admixture of either counter-streaming flux or a nearly isotropic component.

The spectral ratio $N_{\mathrm{p}}(>833 \mathrm{MeV}) / N_{\mathrm{p}}(>500 \mathrm{MeV})$ suggested by the fitted spectrum of interplanetary protons is shown in the lower panel of Figure 2. The two upper panels of Figure 3 show parameters of the proton angular distribution-the anisotropy axis direction, the angle it makes with the IMF, and the pitchangle distribution width $\sigma_{1}$. In the late phase of the event, solar protons arrive from the southwest, at a remarkably large angle to the magnetic field. After 17:10 UT, the proton spectrum becomes a simple power-law spectrum $(\delta \gamma=0)$, while the pitch-angle distribution widens (also Table 2 of Mishev et al. 2018). This is the GLE's delayed component, in contrast to the preceding, prompt component with a steepening spectrum, $\delta \gamma \approx 0.7$, and a narrower pitch-angle distribution. As can be seen from the bottom panel of Figure 2, the spectral ratio of the delayed component decreases logarithmically with time, $N_{\mathrm{p}}(>833 \mathrm{MeV}) / N_{\mathrm{p}}(>500 \mathrm{MeV})=b-a \ln t$ (gray line), which may be one more distinctive property of a delayed component.

Using the empirical fits of the proton pitch-angle distributions, we have calculated the observed net flux of the interplanetary protons and its component perpendicular to the IMF line, with the IMF data described in Section 3. The profile of the highenergy $\gamma$-ray flux produced by protons interacting at the Sun and the flux profiles of high-energy protons near the Earth are compared in the lower panel of Figure 3. While the $\gamma$-ray profile and the interplanetary proton profiles do not coincide, there are clear correspondences between them. We particularly note the coincidence of the maxima of the Phase $3 \gamma$-ray emission and the interplanetary proton flux across the magnetic field.

\section{Solar and Interplanetary Magnetic Environment}

The 2017 September $10 \gamma$-ray flare was bright enough for a time-resolved localization study of the high-energy $\gamma$-ray emission (Omodei et al. 2018, their Figure 3). In our Figure 4, we show the emission localization only for the event's extended phase, Phase 3. It is plotted over the composite image of solar corona, which includes frames from SoHO/LASCO (Brueckner et al. 1995), the Mk4 coronameter of the Mauna Loa Solar Observatory (MLSO; Elmore et al. 2003), and the Sun Earth Connection Coronal and Heliospheric Investigation on Solar Terrestrial Relations Observatory (STEREO/SECCHI; Howard et al. 2008). A prominent feature here is the clearly seen current sheet trailing the CME, with the inverted Y-shaped structure at the sheet's base remarkably situated within the localization area of the $\gamma$-ray source. Note also the magnetic tubes extending from a vicinity of the $\gamma$-ray source to the southwest.

To test the magnetic connectivity of the flare region to Earth, we have performed data-driven, global magnetohydrodynamics simulations of the CME associated with the 2017 September 10 flare using the Alfvén Wave Solar Model (Sokolov et al. 2013; van der Holst et al. 2014), which was previously used to model the 2014 September 1 Fermi behind-the-limb event (Jin et al. 2018). In the case of the 2017 September 10 event, there was a significant number of pre-existing open field lines in the source active region. Figure 5 shows the simulated MHD structures in an early stage of the eruption. The Earth is connected to the CME-driven shock at its eastern flank (by some green lines through the area outlined by the orange dashed contour). Note also that some of the open field lines come from the flare area through the nose of the CME-driven shock and further extend to the interplanetary medium, passing to the west and southwest of the Earth (yellow lines).

Figure 6 provides an overview of the solar wind measurements near the Earth's orbit. The magnetic field is measured on 


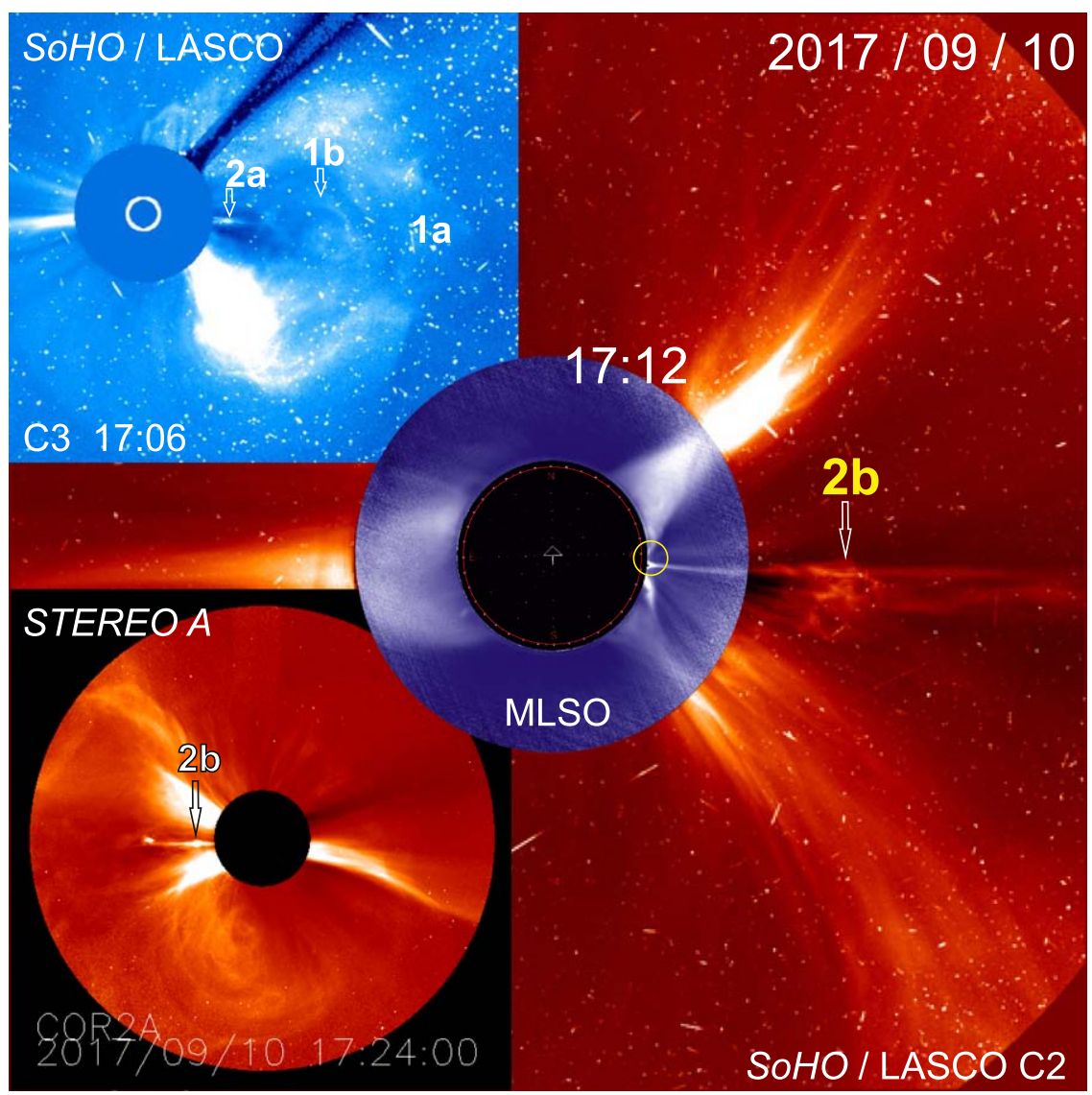

Figure 4. Composite white-light image of the 2017 September $10 \mathrm{CME}$ from spaceborne coronagraphs on SoHO and STEREO and from the Mk4 Coronameter of MLSO. The yellow circle at the solar west limb is the 95\%-confidence-level localization error of the high-energy $\gamma$-ray source at the maximum of Phase 3 emission, 19:03 UT-19:39 UT (Omodei et al. 2018). The insert in the upper left corner shows the CME location at the beginning of Phase 3 (SoHO/LASCO C3): labeled are the CME nose area (1a), the magnetic flux rope (1b), and a jet along the trailing current sheet (2a). The insert in the lower left corner is from STEREO A/COR2 located $128^{\circ}$ east of $\mathrm{SoHO}$, showing another jet along the CME-trailing current sheet (2b), which is also seen in the SoHO/LASCO C2 frame.

board the Advanced Composition Explorer with the Magnetometer instrument (ACE/MAG; Smith et al. 1998) and the solar wind plasma measurements are done with the Solar Wind Electron, Proton, and Alpha Monitor (ACE/SWEPAM; McComas 1998). The spacecraft is orbiting close to the L1 Lagrangian point. For use with the NM network data in Figure 3, the $A C E$ data timing was shifted for the solar wind transit time from $A C E$ to Earth, +50 minutes for the 2017 September 10 case. A series of CMEs at the beginning of 2017 September significantly modified the interplanetary environment for solar particle transport (Guo et al. 2018; Luhmann et al. 2018). The SEP event and GLE 72 were observed inside the interplanetary extension (an ICME) of a CME from a solar eruption on September 6 at 12:24 UT. The estimated start and end times of the ICME passage are based on the plasma and magnetic field observations (see Cane \& Richardson 2003, for additional information on the methods used). The ICME shows evidence of a rotation in the field direction and helium-rich material inside, but lacks an enhanced magnetic field. We note strong fluctuations of the magnetic field direction at the Larmor radius scale of GLE-producing protons, with the amplitude $2|\Delta\langle\lambda\rangle| \sim 30^{\circ}$ (Figure 6, middle panel; the considered fluctuations' timescale is $R_{\mathrm{L}} / U \approx 28$ minutes, where $R_{\mathrm{L}}$ is the gyroradius of a $1 \mathrm{GV}$ proton and $U$ is the solar wind speed). Such IMF irregularities may alter the proton scattering in solar wind compared to the conventional scattering by weak plasma waves. The fluctuations are especially strong near the ICME edges.

\section{Interplanetary Transport Model}

Particle propagation from near the Sun to the Earth's orbit depends on the large-scale IMF structure and scattering conditions. Both factors are highly variable and not completely understood. Our model for analysis of the 2017 September 10 event accounts for the presence of ICME and significant flux of high-energy protons across the IMF lines.

The IMF model employed for the particle transport simulations is shown in Figure 7. It comprises a set of closed magnetic field lines (ICME) and the surrounding solar wind with an open magnetic field. The model is simplified and twodimensional but accounts for relative locations and angular sizes of the relevant 3D structures deduced from the solar corona imaging, MHD modeling results, and in situ observations of the ICME passage. The model parameters can be easily varied for a parametric study. We have compared three IMF models: our basic model, Model 1, with the GLE onset detected inside the $100^{\circ}$-wide ICME; Model 2, with the Earth being at the GLE onset time already $7^{\circ}$ of heliolongitude behind the ICME; and as a reference, Model 3, with magnetic field lines open also in the ICME sector.

The mean-free path for the SEP transport along the IMF lines $\left(\Lambda_{\|}\right)$may be not small compared with the distance to the 


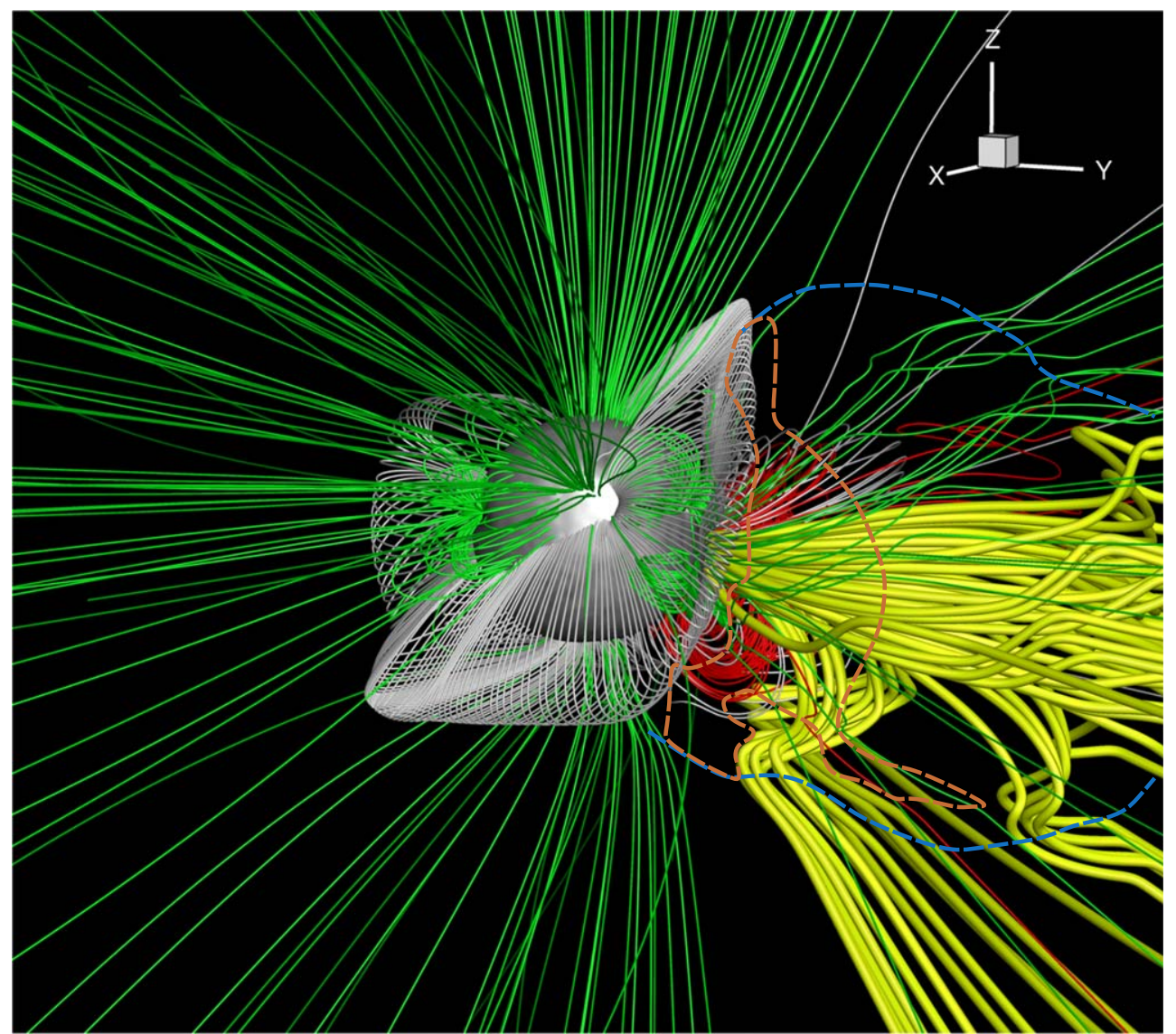

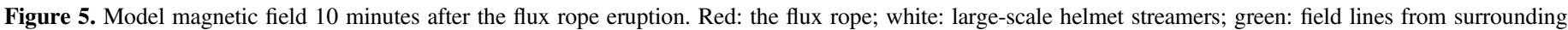

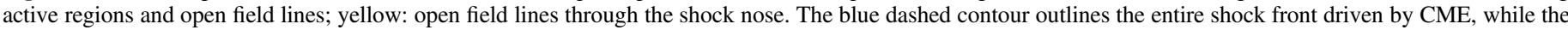
orange dashed contour outlines only the highly compressed area of the shock (compression ratio >4) at the shock's eastern flank partly connected to the Earth.

source, $\Lambda_{\|} \gtrsim 1$ au. For this reason, transport of high-energy protons along the curvilinear coordinate $\xi$ (Figure 7) is considered in the framework of focused transport, which is applicable for both small and large values of $\Lambda_{\|}$(e.g., Toptygin 1985). The classic model of focused transport assumes that energetic particles are scattered off by the weak turbulence in the solar wind plasma, which causes a smallangle (differential) scattering and correspondingly, a pitchangle diffusion in the particle phase space (e.g., Earl 1976). The small-angle scattering often explains the observed SEP flux and anisotropy profiles. However, the GLE observations also reveal an isotropic component of solar high-energy protons rising early in the event. That can be explained by the large-angle (integral) scattering that operates in the high-energy range in addition to the classic pitch-angle diffusion (Kocharov et al. 2017). The large-angle scattering can be caused by strong fluctuations of the magnetic field direction at the Larmor radius scale of relativistic protons, like the fluctuation shown in the middle panel of Figure 6 . The processes considered here for the small-angle scattering and the large-angle scattering are identical to the anisotropic small-angle scattering (AAS) and the small time-step isotropization (SSI) by Kocharov et al. (1998; see also Appendix C of Kocharov et al. 2017).

The scattering rates are parameterized by the values of specific mean-free paths - the mean-free path for the smallangle scattering, $\Lambda_{\mathrm{A}}$, and the mean-free path for the isotropization, $\Lambda_{\mathrm{I}}$. Since no signatures of large-angle scattering have been found so far in the observed pitch-angle distributions of deka-MeV protons, their scattering is modeled with the AAS process only, with rigidity dependence according to the standard quasilinear theory at the Kolmogorov turbulence spectrum (e.g., Kunow et al. 1991). Possible energy dependence of the SSI process within the GLE energy range $(0.4-4 \mathrm{GeV})$ is neglected. Each mean-free path linearly increases with distance from the Sun: $\Lambda_{\mathrm{A}(\mathrm{I})}(\xi) \propto \xi$, except close to the Sun: $\Lambda_{\mathrm{A}(\mathrm{I})}(\xi<0.1 \mathrm{au})=\Lambda_{\mathrm{A}(\mathrm{I})}(0.1 \mathrm{au})$. In what follows, all mean-free path values are given for a $1 \mathrm{GeV}$ proton at the Earth's orbit.

Transport of energetic particles across the IMF lines is typically described as diffusion, with in-depth research showing that the initial cross-field spreading is non-diffusive propagation along meandering field lines (Laitinen et al. 2013). Laitinen \& Dalla (2017) found that the particles begin to decouple from their field lines on timescales of about parallel scattering time, $\tau_{\|} \sim \Lambda_{\|} / v$, and are completely decoupled in $\sim 10 \tau_{\|}$(excluding parallel propagation time effects, Laitinen et al. 2017). In our modeling, a GLE component responsible for the cross-field flux will be registered typically 20 minutes after its injection at the Sun, which is much larger than $\tau_{\|}$for the $\approx 0.5$ au mean-free paths required by the proton flux anisotropy observed in GLE 72 (see below). Under these conditions, we assume that the SEP cross-field propagation has reached its diffusive regime, and model the cross-field propagation as cross-field diffusion.

Perpendicular diffusion is simulated in a local cylindrical coordinate system with the origin situated at the center of 

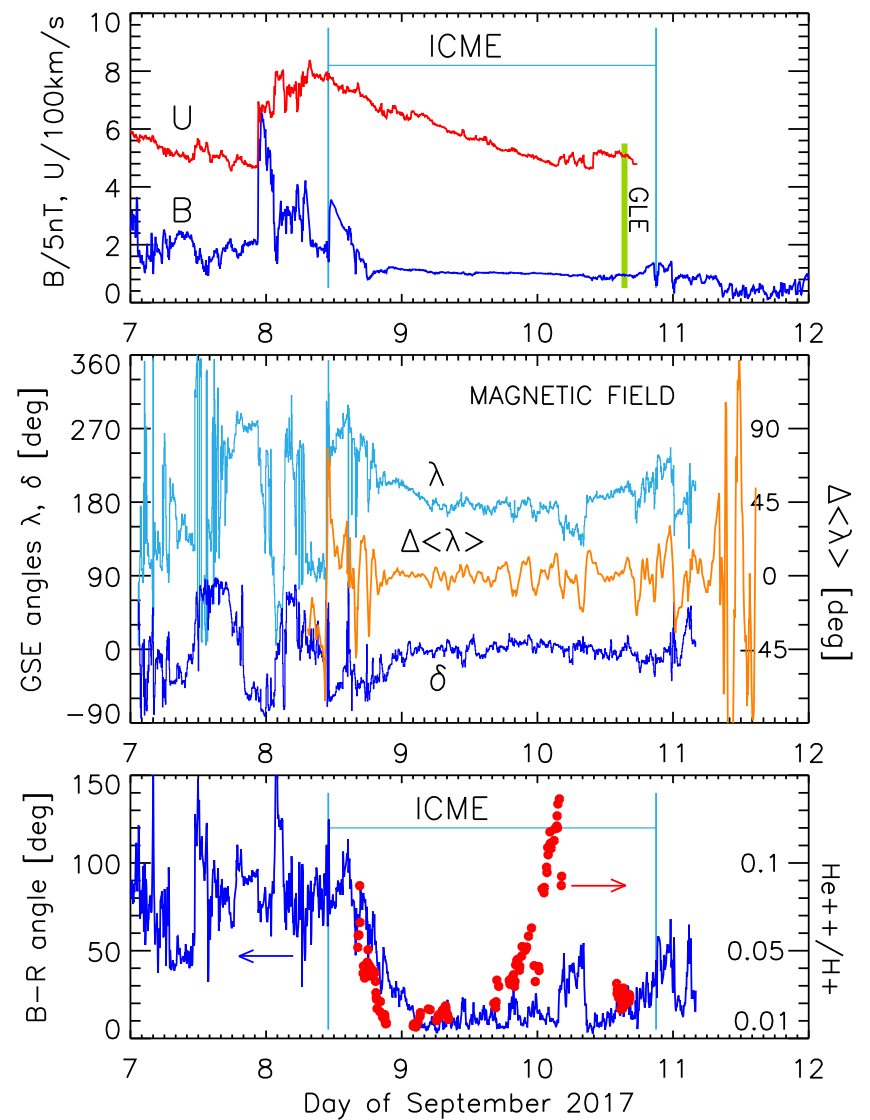

Figure 6. In situ plasma measurements of the passage of interplanetary extension of the 2017 September 6 LASCO CME. Upper panel: magnetic field intensity, $B$, and plasma speed, $U$ (ACE/MAG and $A C E /$ SWEPAM, respectively). Middle panel: the magnetic field angles in the GSE coordinate system, $\lambda$ and $\delta ; \Delta\langle\lambda\rangle$ is a deviation of the gliding 28 minute average value of the IMF azimuthal angle from its 280 minute average value, which is representative of the IMF direction fluctuations at the Larmor radius scale of $\sim 1 \mathrm{GV}$ proton. Lower panel: angle between the magnetic field vector and the radial, Sun-Earth direction (curve; left axis) and the plasma helium-to-proton abundance ratio (points; right axis).

curvature of the current segment of the IMF line (the radial coordinate $\rho$ in Figure 7). The corresponding part of the transport equation for the volumetric number density of energetic particles, $F$, is of the form (e.g., Kocharov et al. 2015b, Equation (8) therein)

$$
\left(\frac{\partial F}{\partial t}\right)_{\perp}=\nabla_{\perp} \cdot D_{\perp} \nabla_{\perp} F
$$

We recast this equation in respect to the distribution over the concentric ring sectors, $f=F \rho$ :

$$
\frac{\partial f}{\partial t}=-\frac{\partial}{\partial \rho}\left[\left(\frac{D_{\perp}}{\rho}+\frac{\partial D_{\perp}}{\partial \rho}\right) f\right]+\frac{1}{2} \frac{\partial^{2}}{\partial \rho^{2}}\left(2 D_{\perp} f\right) .
$$

This Fokker-Plank equation can be expressed as a stochastic differential equation to be solved by time-stepping the progress of individual Monte Carlo particles (e.g., Fletcher 1994). It is very similar to stochastic simulations of the focused transport along the coordinate $\xi$, but the accumulated distribution functions are still not identical. Simulations of the $\rho$-transport yield the number of particles per unit of azimuthal angle of the local cylindrical coordinate system $(F \rho)$, while the $\xi$-transport operates with the number of particles per unit of magnetic tube

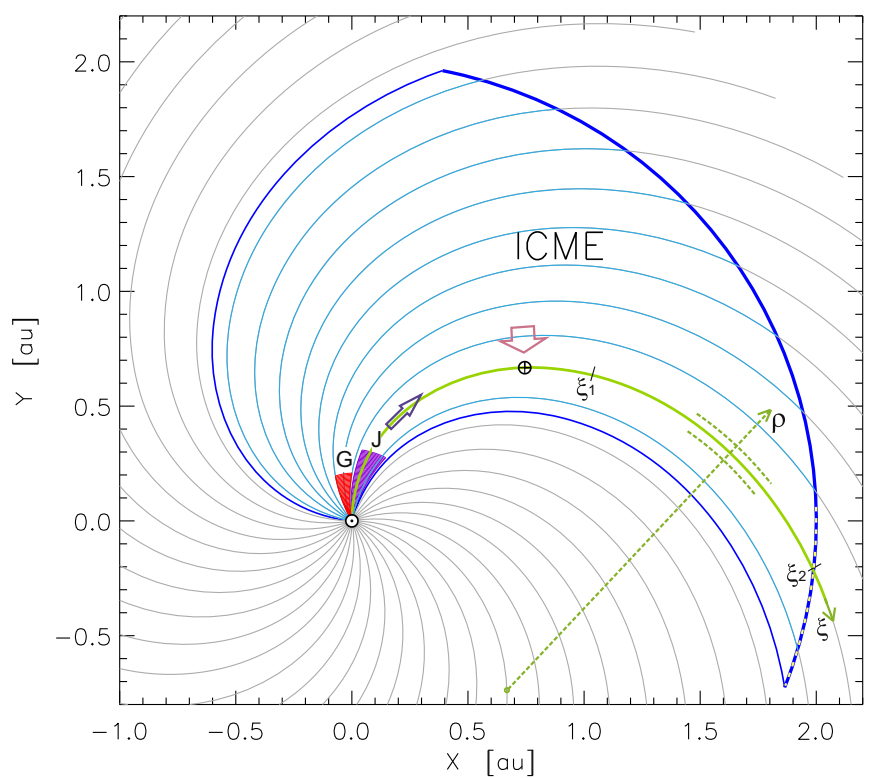

Figure 7. Interplanetary transport model that reproduces both the arrival of the anisotropic particle flux along the magnetic field in the beginning of the event (from Sector $\mathrm{J}$ at the root of the Earth-connected IMF line) and the late, prolonged streaming of high-energy protons across the IMF lines (from Sector $\mathrm{G}$ over the flaring active region near the west limb). The drawing is at scale. The Archimedean spirals of the grid are for the solar wind speed $500 \mathrm{~km} \mathrm{~s}^{-1}$, separated by $10^{\circ}$ in heliolongitude. In the ICME sector, the model magnetic field exponentially increases from $\xi=\xi_{1}$ to $\xi=\xi_{2}$, in total by a factor of 2 . In Models 1 and 2, all simulated particles are bounced back at the ICME top (blue circular arc). In Model 1, ICME spreads in heliolongitude from $-15^{\circ}$ to $+85^{\circ}$ (in respect to the Earth-connected IMF line). Additionally considered are a narrower ICME with an eastern flank shifted westward for $22^{\circ}$ (Model 2), so that the Earth is in the open IMF sector behind the ICME, and Model 3, in which high-energy protons can freely penetrate the ICME top at all locations.

length $(F / B)$. That difference is accounted for by ascribing to a Monte Carlo particle a specific weight that changes at each step in the perpendicular to magnetic field direction.

Perpendicular diffusion is understood to be caused by the field-line meandering at the time-asymptotic limit, with several theoretical approaches relating the turbulence properties to the diffusion coefficient $D_{\perp}=\frac{1}{3} \Lambda_{\perp} v$ (e.g., Jokipii 1966; Giacalone \& Jokipii 1999; Matthaeus et al. 2003; Shalchi 2010; Ruffolo et al. 2012). Here, we use the perpendicular mean-free path, $\Lambda_{\perp}$, that increases with distance from the Sun: $\Lambda_{\perp} \propto B(\xi)^{-1}$. Nominal Parker magnetic field lines are used as a coordinate grid. A particle changes its nominal field line each time it experiences large-angle scattering (isotropization). In such an event, a particle randomly steps in the direction $\rho$ (Figure 7) according to the current value of the product $D_{\perp} \Delta t$, where $\Delta t$ is the time elapsed since the previous isotropization event. The step $\Delta \rho$ accounts for the deviation of the meandering field line from the nominal field line that has been accumulated between the discreet events of large-angle scattering.

\section{Modeling Results}

High-energy protons are injected at two locations shown in Figure 7-at the root of the Earth-connected IMF line (Source $\mathrm{J}$ ) and above the flaring active region (Source $\mathrm{G}$ ). The time profiles of the sources $J$ and $G$, the mean-free path values for the parallel transport, $\Lambda_{\mathrm{A}}$ and $\Lambda_{\mathrm{I}}$, and the perpendicular diffusion coefficient, are adjusted to fit the SEP data. Figure 8 summarizes the modeling results-the inferred 

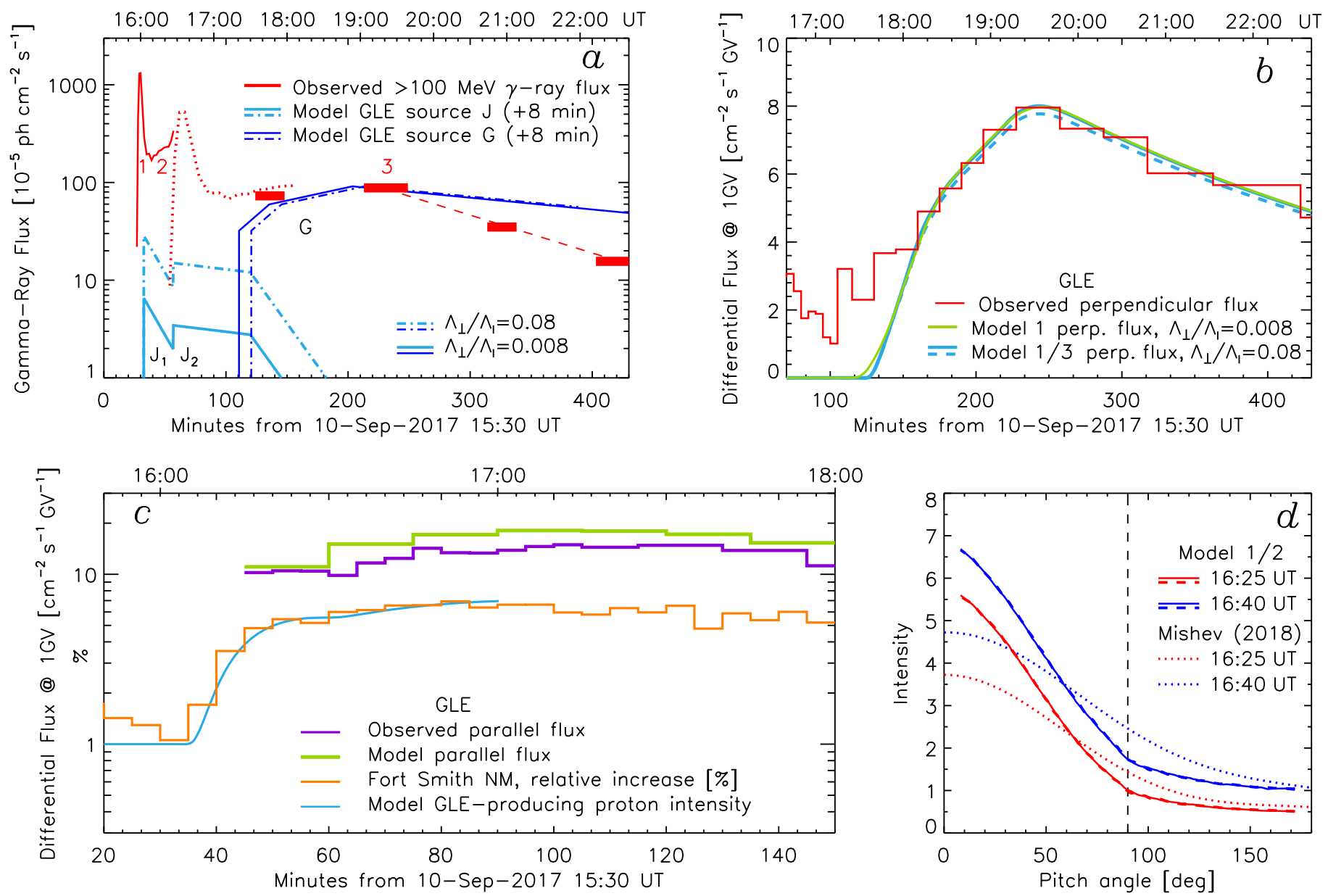

Figure 8. Results of the proton transport modeling. Proton transport is simulated in the IMF of Model 1 with transport parameters $\Lambda_{\mathrm{A}}=5$ au, $\Lambda_{\mathrm{I}}=0.5$ au, and $\Lambda_{\perp} / \Lambda_{\mathrm{I}}$ $=0.008$, unless otherwise specified in a panel. The solar source spectrum is $N_{\mathrm{p}}(E) \propto E^{-4.5}$. The near-Earth profiles are energy-integrated, $E \geqslant 0.5 \mathrm{GeV}$. (a) Injection profile of interplanetary protons (sources $\mathbf{J}$ and $\mathbf{G}$ ) compared with the observed $\gamma$-ray profile (red solid curve and bars). The dotted red line is a hypothetical interpolation between the observed parts of the $\gamma$-ray profile (Section 6). The entire proton source (J+G) is normalized to the peak of Phase $3 \gamma$-ray emission, at either value of $\Lambda_{\perp}$ (without such renormalization, the magnitude of Source G depends on $\Lambda_{\perp}$, while Source $J$ almost does not change). (b) Observed flux of the interplanetary protons across the IMF line and the corresponding model flux at two possible values of the perpendicular diffusion coefficient (dashed line is for Model 3). In the case of $\Lambda_{\perp}=0.08 \Lambda_{\mathrm{I}}$, the magnitude of Source G is $0.23 \times$ the Source G magnitude of the case $\Lambda_{\perp}=0.008 \Lambda_{\mathrm{I}}$. (c) Proton flux parallel to the IMF line. Additionally shown is the count-rate profile of the Fort Smith neutron monitor in percents of GCR background, with $99 \%$ of the background subtracted, and the model intensity profile of GLE-producing protons arriving at pitch angles $12^{\circ}-28^{\circ}$. (d) Pitch-angle distribution of interplanetary protons deduced from the NM network data (Mishev et al. 2018, 5 minutes bins) and our model distribution, both in units corresponding to a $\approx 1 \%$ increase of the Fort Smith NM count rate (dashed lines are for Model 2).

SEP source profiles (panel (a)), the proton net flux in the perpendicular to the IMF direction (b), the parallel flux (c), and the proton pitch-angle distributions (panel (d)).

\subsection{Interplanetary Transport Parameters}

Interplanetary transport of SEPs depends on scattering conditions in the solar wind plasma, which should be estimated on an event-by-event basis with use of the SEP data, preferentially the data of particle flux anisotropy (e.g., Kunow et al. 1991). Particle telescopes in space beyond the Earth's magnetosphere, like the High-Energy Detector of the Energetic and Relativistic Nuclei and Electron instrument (ERNE/HED) on $\mathrm{SoHO}$ can provide such data in the deka-MeV range (Torsti et al. 1995) and those data indicate that the proton mean-free path often exceeds 1 au (e.g., Torsti et al. 2004). The $\mathrm{SoHO} /$ ERNE data, however, are not available for 2017 September 10. Another particle instrument, SoHO/EPHIN, possesses good sensitivity and energy resolution, detects particles arriving in a fixed, $83^{\circ}$-wide view cone, and by this expedient, has a capability for assessment of the width of the proton pitch-angle distribution. At the beginning of the 2017 September 10 event, the IMF direction stayed inside the instrument's view cone until 17:20 UT and was $\approx 20^{\circ}$ off the view cone for the next 20 minutes (Figure 1(c)). The latter excursion of the magnetic field allows us to estimate the width of the proton pitch-angle distribution and the corresponding mean-free path value. Another opportunity for estimating the mean-free path was mentioned in Section 2. It is the velocity dispersion of the SEP rise profiles. With two opportunities together, a comparison of the observed SEP profiles with the particle transport simulations accounting for the small-angle scattering, magnetic focusing, and the particle sampling into the EPHIN's view cone, suggests a parallel mean-free path of $30 \mathrm{MeV}$ protons $\Lambda_{\mathrm{A}}(30 \mathrm{MeV})=3 \pm 1$ au. In the framework of the quasilinear theory of the pitch-angle scattering with the Kolmogorov turbulence spectrum, this corresponds to a mean-free path of $1 \mathrm{GeV}$ protons $\Lambda_{\mathrm{A}}(1 \mathrm{GeV})=4-8 \mathrm{au}$, to be refined with the NM network data. 
Proton flux anisotropy data in the $\mathrm{GeV}$ range are available from the NM network (Mishev et al. 2018). Those data indicate an early appearance of an isotropic component of the pitchangle distribution, which in our model is accounted for with the large-angle scattering of relativistic protons with a mean-free path $\Lambda_{\mathrm{I}}$, a process concurrent to the small-angle scattering with mean-free path $\Lambda_{\mathrm{A}}$. Values of $\Lambda_{\mathrm{I}}$ and $\Lambda_{\mathrm{A}}$, along with the time profile of solar Source $J$, are adjusted to fit the proton flux anisotropy data of the NM network in the first phase of the event (Figure $8(\mathrm{~d})$ ). The best-fit values of the specific meanfree paths are $\Lambda_{\mathrm{A}}=5 \mathrm{au}$ and $\Lambda_{\mathrm{I}}=0.5 \mathrm{au}$. The $\Lambda_{\mathrm{I}}$ value provides a sufficient number of backward-propagating protons (pitch angles $\alpha>90^{\circ}$ ), while $\Lambda_{\mathrm{A}}$ provides a necessary steepness of the distribution in the forward direction to fit the Fort Smith data without a similar increase at other stations. Note that the previously inferred, empirical pitch-angle distributions (shown with dotted lines in Figure 8(d)) markedly underestimate the Forth Smith count rates observed in the first phase of the event (before 17:15 UT; Mishev et al. 2018, Figure 9 therein), while the present model fits the Fort Smith data better.

In the extended phase of the event, after 18:00 UT, a contribution of the cross-field transport into the particle flux near the Earth rises significantly, such that the model proton flux becomes essentially dependent on the adopted value of the perpendicular mean-free path $\Lambda_{\perp}$. In Figure 8, we show the modeling results for two values of the perpendicular mean-free path: $\Lambda_{\perp}=0.008 \Lambda_{\mathrm{I}}$ and an order-of-magnitude larger value, $\Lambda_{\perp}=0.08 \Lambda_{\mathrm{I}}$. These values bracket the available theoretical estimates (e.g., Giacalone \& Jokipii 1999).

\subsection{Particle Profiles}

The inferred source profiles of solar high-energy protons are shown in Figure 8(a). An adopted value of the perpendicular mean-free path affects the model perpendicular flux. Correspondingly, given the observational flux, the uncertainty of $\Lambda_{\perp}$ comes to the uncertainty of the Source $G$ magnitude and in its turn, to the relative strength of two sources, $G$ versus $J$. The total numbers of protons injected by Source $\mathbf{J}\left(\mathbf{J}_{1}+\mathbf{J}_{2}\right)$ and Source $\mathrm{G}$ over the entire time of their operation are

$$
\begin{aligned}
N_{\mathrm{J}}(>500 \mathrm{MeV}) & =1.6 \times 10^{30}, \\
N_{\mathrm{G}}(>500 \mathrm{MeV}) & =\chi N_{\mathrm{J}}(>500 \mathrm{MeV}),
\end{aligned}
$$

where $\chi=64$ at $\Lambda_{\perp} / \Lambda_{\mathrm{I}}=0.008$ and $\chi=14$ at $\Lambda_{\perp} / \Lambda_{\mathrm{I}}$ $=0.08$. For these estimates of the source-area-integrated numbers, $N_{\mathrm{J}}(>500 \mathrm{MeV})$ and $N_{\mathrm{G}}(>500 \mathrm{MeV})$, we have assumed that the surface area of each of two near-Sun sources is $0.1 R_{\odot}^{2}$, which corresponds to the heliocentric angular size of $20^{\circ}$ (Figure 7). That is about the size of the yellow circle in Figure 4. Somewhat smaller or larger source areas could not be ruled out either and correspondingly may affect the interplanetary proton number estimates.

The time profile of the proton flux perpendicular to the IMF is shown in Figure 8(b). The observed flux is calculated with the $1 \mathrm{GV}$ proton intensities deduced from the NM network data by Mishev et al. (2018) and the 100 minute average IMF directions measured by $A C E / \mathrm{MAG}$. The model flux is simulated with the ICME Model 1 at two possible values of the perpendicular diffusion coefficient. We also show the result of modeling with ICME Model 3, in which protons are not bounced at the top of the ICME (dashed line). The effect of the
IMF topology turns out to be weak because the ICME top is already more than 1 au behind the Earth.

The parallel flux of the GLE-producing protons is shown in Figure $8(\mathrm{c})$. In order to refine the injection onset profile, we have additionally fitted the 5 minute count-rate profile of the Fort Smith NM. The 5 minute width of the bins implies corresponding accuracy of the inferred timing of the leading front of Source $J_{1}$ in panel (a). Our present theoretical model suggests a somewhat steeper pitch-angle distribution in the forward hemisphere as compared to the empirical fit by Mishev et al. (2018). For this reason, the model parallel flux shown in panel (c) exceeds the parallel flux estimated with the previous empirical fit to the NM network data (observed flux).

\section{Discussion}

We have modeled the interplanetary transport of high-energy solar protons in a hybrid model that treats particle transport along the IMF lines in the focused transport approximation, while the cross-field transport is modeled in a diffusion approximation. Such a hybrid model is a straightforward modification of our previous models of focused transport (Kocharov et al. 1998, 2005, 2009). As compared to the hybrid model by Dröge et al. (2014), we do not assume conservation of the particle pitch-angle in the event of the particle decoupling from the magnetic field line. In addition, our treatment of perpendicular transport also accounts for the curvature of magnetic field lines. The SEP transport parameters are estimated on an event-by-event basis. With the available data, we estimate the $\mathrm{GeV}$ proton mean-free-path values in the 2017 September 10 event to be: $\Lambda_{\mathrm{I}}=0.5 \mathrm{au}$ for the largeangle/integral scattering and $\Lambda_{\mathrm{A}}=5 \mathrm{au}$ for the small-angle/ differential scattering. A large uncertainty still remains in the assessment of the cross-field transport rate.

Share et al. (2018) reviewed observations of the late-phase $\gamma$-ray emission (LPGRE) since the Solar Maximum Mission era. The timescales of the LPGRE range from a fraction of an hour to $8 \mathrm{hr}$, so the LPGRE group would include both Phase 2 and Phase 3 emissions of the 2017 September 10 flare observed by Fermi/ LAT. The number of LPGRE protons $>500 \mathrm{MeV}$ in nine events considered by Share et al. (2018) was estimated to range from $0.0015 \times$ to $0.5 \times$ the number in the accompanying SEP event. In the 2017 September 10 event, the total number of protons injected to the interplanetary medium by Source $G$ over the time interval 18:59:00 UT-19:26:30 UT (solar time) is $N_{\uparrow}(>500 \mathrm{MeV})=1.9 \times 10^{29} \chi$, where $\chi$ depends on the adopted value of the perpendicular diffusion coefficient, like in Equation (3). The number of $>500 \mathrm{MeV}$ protons interacting at the Sun over the same time interval $N_{\downarrow}(>500 \mathrm{MeV})=3 \times 10^{28}$. Correspondingly, the number ratio of protons interacting at the Sun in Phase 3 of the $\gamma$-ray production and protons injected to the interplanetary space by Source $G$ is

$$
\frac{N_{\downarrow}(>500 \mathrm{MeV})}{N_{\uparrow}(>500 \mathrm{MeV})}=\frac{0.16}{\chi},
$$

where $\chi=15-60$. The interplanetary proton number exceeds the number of interacting protons, even though we have a large uncertainty in the perpendicular transport parameters. In the preceding phase (Phase $2 / \mathrm{J}$ ), the ratio of $\gamma$-rayproducing protons to GLE-producing protons is about $\chi \times$ the ratio in Phase $3 / \mathrm{G}$ (Figure $8(\mathrm{a})$ ), such that $\left(N_{\downarrow} / N_{\uparrow}\right)_{2 / \mathrm{J}} \sim 0.2$. Our estimates of $N_{\downarrow} / N_{\uparrow}$ are in the range reported by 
Share et al. (2018) for other events. Recently, de Nolfo et al. (2019) analyzed 14 LPGRE events and found that the interplanetary proton number is poorly correlated with the number of protons interacting at the Sun. Our case study and modeling of the 2017 September 10 event suggest that an estimate of the $N_{\downarrow} / N_{\uparrow}$ ratio can span a few orders of magnitude due to the variation of many factors, including, in particular, the relative contribution of the particle components $\mathrm{J}$ and $\mathrm{G}$, magnetic connectivity, and interplanetary transport conditions, even when both proton populations in each particular event originate from a common source.

With a given response function of neutron monitors and count statistics of GLE 72, the proton spectrum deduced from the NM network data refers to the energy range $\sim 400 \mathrm{MeV}-$ $4 \mathrm{GeV}$. The $\mathrm{GeV}$ proton spectrum deduced from the NM data for 17:30 UT-17:55 UT is as steep as $\sim E^{-5.5}$. The energy spectrum of interplanetary protons in the 2017 September 10 event was measured also in the deka-to-hecto-MeV range by spaceborne instruments (Matthiä et al. 2018; Bruno et al. 2019), in particular by SoHO/EPHIN. The EPHIN instrument on $\mathrm{SoHO}$ was originally optimized to measure proton and helium isotope spectra up to $50 \mathrm{MeV}$ nucl $^{-1}$, but a capability of determining the energy spectra up to $\sim 600 \mathrm{MeV} \mathrm{nucl}^{-1}$ also exists (Kühl et al. 2015; Kühl \& Heber 2019). During 16:00 UT-21:00 UT, the $60-600 \mathrm{MeV}$ proton spectrum from EPHIN does not change much; it is nearly as hard as $\sim E^{-2.7}$ around $300 \mathrm{MeV}$. The energy range of interacting protons for the production of pions falls at the boundary between the EPHINobserved and NM-observed energy ranges of the interplanetary protons. The interacting proton spectrum above $300 \mathrm{MeV}$ at 17:40 UT-17:50 UT is estimated as $\sim E^{-3.2}$. It is softer than the EPHIN-observed spectrum of interplanetary protons around $300 \mathrm{MeV}, \sim E^{-2.7}$, and harder than the NM-observed spectrum of the $\gtrsim 1 \mathrm{GeV}$ protons, $\sim E^{-5.5}$. Possible bending of the proton spectra around $500 \mathrm{MeV}$ adds complexity to their comparison, so a further analysis is required. Note that bent spectra of highenergy protons were previously found in many other events (e.g., Kocharov et al. 1996; Torsti et al. 1996; Tylka et al. 2010).

Evidence of the presence of two different components of high-energy particles in a single GLE, a prompt component and a delayed component, were revealed in many events (e.g., Miroshnichenko et al. 1995; McCracken et al. 2008). This is the case also for the 2017 September 10 event. The GLE starts with the prompt component that streams along the IMF line with a relatively narrow pitch-angle distribution, while the proton spectrum markedly steepens with rigidity within the NM rigidity range. After 17:10 UT we observe a transition to the delayed component: the spectrum becomes a single power law over the entire rigidity range and angular distribution of the near-Earth solar protons widens. In our modeling, a source of the prompt component is situated at the root of the Earthconnected IMF line $\left(\mathrm{J}_{1}\right.$ and partly $\left.\mathrm{J}_{2}\right)$, while the delayed component arrives largely across the magnetic field lines from the source situated above the flaring region near the solar western limb (Source G; Figures 7 and 8).

SEPs originating from different sources may have different ion abundances. In the impulsive-gradual classification of SEP event, a low helium-to-proton ratio, $\mathrm{He} / \mathrm{p} \sim 0.001-0.01$, is indicative of a gradual events, while high values, $\mathrm{He} / \mathrm{p} \gtrsim 0.1$, define impulsive events (e.g., Kocharov et al. 1983; Cane et al. 1986; Reames 1995b; Cliver 1996). A helium-rich abundance

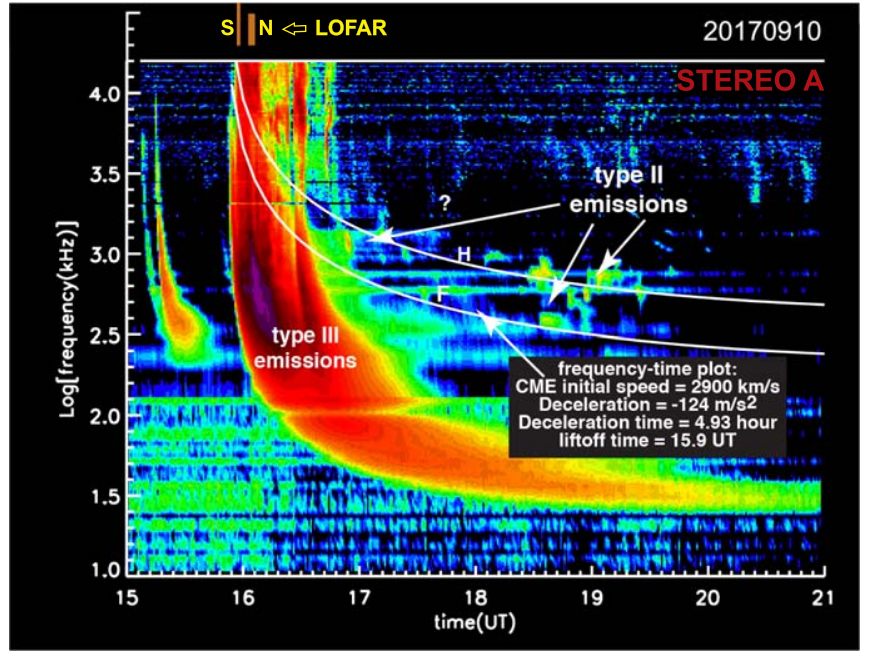

Figure 9. Dynamic low-frequency radio spectrum detected by STEREO A/ WAVES on 2017 September 10 (Bougeret et al. 2009). Pair curves F and H (fundamental and harmonic) show one of few possible kinematic fits to the type II emission that assumes emission from the CME nose as observed on STEREO A and the density model by Saito et al. (1977) with an enhanced solar wind density amounting to $35 \mathrm{~cm}^{-3}$ near the Earth's orbit (M. Reiner 2018, private communication). Additionally shown are locations of the LOFARobserved shock-associated emissions: a herringbone structure (S) and a fragment of the type II fundamental lane $(\mathrm{N})$, which originate from the CME's southern and northern flanks, respectively (Group 1 and Group 3 in Figures 1(c) and 5(a) by Morosan et al. 2019).

was previously reported for GLE 70 on 2006 December 13 in the energy range extending from $\sim 30 \mathrm{MeV}$ to $\sim 2 \mathrm{GeV}$ (Adriani et al. 2011; Kocharov et al. 2015a). In the case of the 2017 September 10 event, the helium abundance was measured by $\mathrm{SoHO} / \mathrm{EPHIN}$ in the deka-to-MeV range to be $\mathrm{He} / \mathrm{p}=0.08$ (Figure 1(f)), which is an impulsive composition, expected to originate from the flare site, not from the CMEbow shock in the solar wind. On their way from the solar source to the interplanetary space, the helium-rich SEPs should have penetrated the shock driven by CME at its eastern flank connected to the Earth (Figure 5). The shock, however, was not an obstacle when the helium-rich SEPs arrived at the beginning of the 2017 September 10 event. The SEP transit through the shock may be possible via fast transport channels/quiet magnetic tubes penetrating the shock (similar to model by Kocharov et al. 2014). Such channels could also provide a way for the back precipitation of the shock-accelerated particles to the Sun to produce there, via nuclear interactions, a variety of secondary emissions (Kocharov et al. 2015b).

The prompt component of the 2017 September 10 GLE is associated with a series of late type III radio bursts persisting until 16:55 UT (Figure 9), possibly indicative of the opening of magnetic traps from which the prompt component could be released directly to the Earth-connected IMF lines (see Cane et al. 2002). Such an association was observed also in a number of other GLE events (so-called GLEs of type J; Kocharov et al. 2018), in particular in GLE 67 on 2003 November 2, which was similar to the 2017 September 10 event in terms of associated coronal and CME structures (our present Figure 4 versus Figure 11 by Kocharov et al. 2017). However, now we focus on the prolonged, delayed components of interplanetary protons and on the interacting protons generating the prolonged $\gamma$-ray bursts. 
There is a gap in the Fermi-LAT-data between the end of the observation period $2 \mathrm{~b}$ and the first observed part of period 3 (Figure 2). The $2 \mathrm{~b}$ emission likely is the leading front of a $\gamma$-ray burst that is similar to the microwave burst observed at that time by EOVSA (Figure 1 by Gary et al. 2018, our burst mw2). For illustration purposes, in Figure 8(a) we have filled the gap in $\gamma$-ray data with a hypothetic profile based on the idea by Cliver (1996), who proposed that the pion-rich phases of large flares may be similar to those occurring earlier in the flare, the main difference being the greater height of the accelerated region and presence of previously accelerated particles. Assuming such a similarity of processes at different scales, we have used the observed impulsive-gradual pattern of period $1-2 \mathrm{a}$ to extrapolate the $\gamma$-ray profile from period $2 \mathrm{~b}$ onward. The observed profile $1-2 \mathrm{a}$ has been shifted in time and stretched by a factor of 4 , and also renormalized by a factor of 0.4 so that the scaled profile fits with both the observed rise phase of the $\gamma$-ray burst $2 b$ and the decay phase of the 25-100 keV X-ray burst observed at 16:40 UT-16:45 UT by the Reuven Ramaty High-Energy Solar Spectroscopic Imager (Lin et al. 2002). This extrapolation matches remarkably well with the observed part of Phase 3 emission.

The $\gamma$-ray burst of Phase 3 is localized near the western limb within the area indicated with the yellow circle in Figure 4, which includes high loops at the base of a bright ray that is the current sheet trailing the CME. The Phase $3 \gamma$-ray emission originates from a compact source but changes in time very slowly, with an e-folding time of $1.8 \mathrm{hr}$ (Omodei et al. 2018). Such a simultaneous involvement of a small spatial scale and a large timescale indirectly supports the idea that a thin and long object like the plasma sheet trailing the CME may be involved in production of the Phase $3 \gamma$-ray emission. Current sheets trailing the CMEs in other events were studied in detail by Lin et al. (2004) and Webb \& Vourlidas (2016).

Thanks to EOVSA observations, we have high-resolution images of the associated microwave sources, including the latephase burst mw2 (Figure 2 by Gary et al. 2018). The mw2 burst arises from two sources located at the sides of the rising bright loops seen in the $193 \AA$ images by the Atmospheric Imaging Assembly on the Solar Dynamics Observatory (SDO/AIA; Lemen et al. 2012) and also in the white-light images from MLSO beneath the current sheet (our Figure 4 and Figure 4(a) by Cheng et al. 2018). The onset of the mw2 burst at 16:26 UT (Figure 1 by Gary et al. 2018) exactly coincides with the onset of the $\gamma$-ray burst $2 \mathrm{~b}$ (our Figure 2 or Table 1 by Omodei et al. 2018). For these reasons, the microwave source mw 2 and the source of the $\gamma$-ray burst $2 \mathrm{~b}$ should be situated close to each other at/around the high loops beneath the CME current sheet.

The bright ray seen in EUV and white-light images after 16:00 UT is interpreted as a current sheet that formed in the wake of CME. The current sheet of the 2017 September 10 CME was thicker than is typically observed, and had a high emission measure, and for this reason, it was well investigated. Warren et al. (2018), using observations from the EUV Imaging Spectrometer (Culhane et al. 2007) on board Hinode (Kosugi et al. 2007) and AIA on SDO, found that plasma in the current sheet reaches temperatures of about $20 \mathrm{MK}$. The highest temperatures occur at the base of the current sheet, in the region near the top of the post-flare loop arcade, and Fe line emission indicates a coronal composition and suggests that the current sheet is formed by the heating of plasma already in the corona. Cheng et al. (2018) reported the fragmented and turbulent nature of magnetic reconnection in the super-hot plasma current sheet. Two oppositely directed outflow jets are intermittently expelled out of the fragmenting current sheet. The speeds of the sunward outflow jets typically are within $100-600 \mathrm{~km} \mathrm{~s}^{-1}$ (Figure 3 by Cheng et al. 2018). The MLSO $\mathrm{K}$-Cor instrument provides white-light images of the extended current sheet at its later phase, starting at 17:12 UT (the frame embedded in Figure 4) and continuing through 20:10 UT. By chance, the MLSO observation period nearly coincides with the maximum phase of the high-energy $\gamma$-ray burst 3 and the brightening of the low-frequency type II radio emission observed on STEREO A (Figure 9). Cheng et al. (2018) count in the MLSO frames eight anti-sunward moving blobs with velocities up to $\sim 1200 \mathrm{~km} \mathrm{~s}^{-1}$. Some outflow jets are seen moving farther away from the Sun in images from $\mathrm{SoHO}$ and STEREO coronagraphs (like the two jets labeled $2 \mathrm{a}$ and $2 \mathrm{~b}$ in Figure 4). Using both SoHO and STEREO A observations, we estimate the non-projected velocity of jet $2 \mathrm{~b}$ to be about $1600 \mathrm{~km} \mathrm{~s}^{-1}$. The extrapolated launch time, $\sim 16: 42 \mathrm{UT}$, is within the microwave enhancement period mw2, while the low corona observations at MLSO did not start by that time.

The idea of post-impulsive phase acceleration of high-energy protons in solar corona has been discussed for years, along with particle acceleration in the CME-driven shocks in solar wind. In particular, Klein et al. (2001) reported observational results of the 2000 July 14 eruption (GLE 59) that indicated that the relativistic protons in that event were accelerated during the magnetic field reconfiguration at heights between $0.1 R_{\odot}$ and $1 R_{\odot}$ above the photosphere, and not in the flaring active region or at the bow shock of the CME. More recently, the trailing current sheets of CMEs have been proposed to accelerate electrons (Pick et al. 2005). Even though relativistic electrons are observed in the 2017 September 10 event (Figure 1(d)), we concentrate now on acceleration of high-energy protons.

Different magnetohydrodynamic (MHD) motions are typically considered for acceleration of protons and heavier ions, and such motions are observed on 2017 September 10, including the CME-bow shock in solar wind, and, much closer to the Sun, the repetitive jets and turbulence in the plasma sheet trailing the CME. The launch of jet $2 \mathrm{~b}$ may be associated with microwave burst mw2 that apparently triggered the Phase $3 / \mathrm{G}$ proton production (Figure 4). In the frame of the coronal acceleration concept, the hard-spectrum protons producing the Phase $3 \gamma$-ray emission could be accelerated on closed magnetic field lines below the CME current sheet by MHD turbulence and local shocks caused by fragmented magnetic reconnection and jets expelled from the plasma sheet. Accelerated protons simultaneously precipitate into the chromosphere and escape into the solar wind.

A traditional candidate for high-energy proton production in gradual (phase of) SEP events is the CME-bow shock (Reames 1999). The observed coincidence of the type II radio enhancement (Figure 9) and the $\gamma$-ray emission of Phase 3 (Figure 8(a)) seems to support acceleration of interplanetary protons by the CME-driven shock. Such a model was tested by Kocharov et al. (2015b). However, the height of the model CME was much smaller than the CME height in the 2017 September 10 event at the time of the Phase $3 \gamma$-ray emission. On the other hand, the low-frequency type II source and the proton acceleration site may be located not at the CME nose but closer to the Sun, e.g., at the flank of the CME. Radio signatures of the CME-driven shock in the deka-MHz band, 
including herringbone structures and type II lanes, were observed on 2017 September 10 by the Low Frequency Array (LOFAR; Morosan et al. 2019). They appear at the CME flanks, first in the southwest and then in the northwest (Group 1-2 and Group 3 in Figure 5(a) by Morosan et al. 2019). If protons were accelerated at a fraction of the CME height, they would have a higher chance of returning to the Sun, compared to returning from the CME nose.

A hybrid scenario that includes episodes of reacceleration of flare-originating particles should be also considered. Highenergy ions of GLE 70 (2006 December 13) were observed by both neutron monitors on the ground (in particular, Mishev \& Usoskin 2016) and the Payload for Matter-Antimatter Exploration and Light Nuclei Astrophysics in space (PAMELA; Adriani et al. 2011). The PAMELA data indicate that the helium-to-proton abundance ratio in the GV rigidity range was high (impulsive composition) and did not change at the transition from the GLE's prompt component, observed from 02:50 UT until $\approx 03: 50$ UT on 2006 December 13, to the delayed component continuing after 03:50 UT through the end of the day, while the energy spectrum shape did change at around 03:50 UT from an exponential shape to a power-law one. This supports the idea that the delayed component in that event was produced by a reacceleration of energetic particles originating from the flaring region.

\section{Conclusion}

The observed time profile of the high-energy $\gamma$-ray emission, produced by protons interacting at the Sun, comprises several parts, including the extremely prolonged, Phase 3 emission. We find a counterpart of the Phase 3 solar protons in the interplanetary protons arriving at the Earth across the IMF lines. Data and modeling support the idea that those two proton populations originate from a common, CME-driven source. The source may be situated either at the CME-bow shock or behind the CME near the CME-trailing current sheet. The latter possibility has not yet received sufficient attention.

We thank the anonymous referee for stimulating comments. This research is supported by the Academy of Finland at University of Oulu (projects 304435/CRIPA-X and 267186) and University of Turku (decision 312357). SoHO is a project of international cooperation between ESA and NASA.

\section{ORCID iDs}

Leon Kocharov (i) https://orcid.org/0000-0002-2077-5618 Melissa Pesce-Rollins (i) https://orcid.org/0000-00031790-8018

Timo Laitinen (1) https://orcid.org/0000-0002-7719-7783

Meng Jin (1) https://orcid.org/0000-0002-9672-3873

Nicola Omodei (i) https://orcid.org/0000-0002-5448-7577

Francesco Longo (1) https://orcid.org/0000-0003-2501-2270

David F. Webb (1) https://orcid.org/0000-0001-5948-559X

Bernd Heber (1) https://orcid.org/0000-0003-0960-5658

Ilya Usoskin (1) https://orcid.org/0000-0001-8227-9081

\section{References}

Ackermann, M., Ajello, M., Albert, A., et al. 2014, ApJ, 787, 15 Ackermann, M., Allafort, A., Baldini, L., et al. 2017, ApJ, 835, 219 Adriani, O., Barbadino, G. C., Bazilevskaya, G. A., et al. 2011, ApJ, 742, 102 Ajello, M. A. A., Allafort, A., Baldini, L., et al. 2014, ApJ, 789, 20
Atwood, W. B., Abdo, A. A., Ackermann, M., et al. 2009, ApJ, 697, 1071 Bougeret, J. L., Goetz, E., Kaiser, M. L., et al. 2009, SSRv, 136, 487

Brueckner, G. E., Howard, R. A., Koomen, M. J., et al. 1995, SoPh, 162 , 357

Bruno, A., Christian, E. R., de Nolfo, G. A., Richardson, I. G., \& Ryan, J. M. 2019, SpWea, 17, 419

Cane, H., Erickson, W. C., \& Prestage, N. P. 2002, JGRA, 107, 1315

Cane, H., \& Richardson, I. G. 2003, JGRA, 108, 1156

Cane, H. V., McGuire, R. E., \& von Rosenvinge, T. T. 1986, ApJ, 301, 448

Cheng, X., Li, Y., Wan, L. F., et al. 2018, ApJ, 866, 64

Cliver, E. W. 1996, in AIP Conf. Proc. 374, High Energy Solar Physics, ed. R. Ramaty, N. Mandzhavidze, \& X.-M. Hua (New York: AIP Press), 45

Culhane, J. L., Harra, L. K., James, A. M., et al. 2007, SoPh, 243, 19

de Nolfo, G. A., Bruno, A., Ryan, J. M., et al. 2019, ApJ, 879, 90

Dröge, W., Kartavykh, Y. Y., Dresing, N., Heber, B., \& Klassen, A. 2014, JGRA, 119, 6074

Earl, J. A. 1976, АpJ, 205, 900

Elmore, D. F., Burkepile, J. T., Darnell, J. A., Lecinski, A. R., \& Stanger, A. L. 2003, Proc. SPIE, 4843, 66

Fletcher, L. 1994, SSRv, 68, 239

Gary, D. E., Chen, B., Dennis, B., et al. 2018, ApJ, 863, 83

Giacalone, J., \& Jokipii, J. R. 1999, ApJ, 520, 204

Gopalswamy, N., Yashiro, S., Mäkelä, P., et al. 2018, ApJL, 863, L39

Guo, J., Dumbović, M., Wimmer-Schweingruber, R. F., et al. 2018, SpWea, 16,1156

Howard, R. A., Mosses, J. D., Vourlidas, A., et al. 2008, SSRv, 136, 67

Hua, X.-M., \& Lingenfelter, R. E. 1987, SoPh, 107, 351

Jiggens, P., Clavie, C., Evans, H., et al. 2019, SpWea, 17, 99

Jin, M., Petrosian, V., Liu, W., et al. 2018, ApJ, 867, 122

Jokipii, J. R. 1966, ApJ, 146, 480

Klein, K.-L., Trottet, G., Lantos, P., \& Delaboudinière, J.-P. 2001, A\&A, 373, 1082

Kocharov, G. E., Kovaltsov, G. A., \& Kocharov, L. G. 1983, Proc. 18th ICRC (Bangalore, India), 4, 105

Kocharov, L., Klassen, A., Valtonen, E., Usoskin, I., \& Ryan, J. M. 2015a, ApJL, 811, L9

Kocharov, L., Kovaltsov, G., Laitinen, T., Mäkelä, P., \& Torsti, J. 1999, ApJ, 521,898

Kocharov, L., Kovaltsov, G. A., Torsti, J., \& Huttunen-Heikinmaa, K. 2005, JGR, 110, A12S03

Kocharov, L., Laitinen, T., Usoskin, I., \& Vainio, R. 2014, ApJL, 787, L21

Kocharov, L., Laitinen, T., Vainio, R., et al. 2015b, ApJ, 806, 80

Kocharov, L., Pizzo, V. J., Odstrcil, D., \& Zwickl, R. D. 2009, JGR, 114 A05102

Kocharov, L., Pohjolainen, S., Mishev, A., et al. 2017, ApJ, 839, 79

Kocharov, L., Pohjolainen, S., Reiner, M., et al. 2018, ApJL, 862, L20

Kocharov, L., Torsti, J., Vainio, R., Kovaltsov, G. A., \& Usoskin, I. G. 1996, SoPh, 169, 181

Kocharov, L., Vainio, R., Kovaltsov, G., \& Torsti, J. 1998, SoPh, 182, 195

Kosugi, T., Matsuzaki, K., Sakao, T., et al. 2007, SoPh, 243, 3

Kühl, P., Dresing, N., Gieseler, J., Heber, B., \& Klassen, A. 2015, Proc. ICRC (The Hague, The Netherlands), 239, 224

Kühl, P., \& Heber, B. 2019, SpWea, 17, 84

Kunow, H., Wibberenz, G., Green, G., Müller-Mellin, R., \& Kallenrode, M.-B 1991, in Physics of Inner Heliosphere II, ed. R. Schwenn \& E. Marsch (Berlin: Springer), 243

Laitinen, T., \& Dalla, S. 2017, ApJ, 834, 127

Laitinen, T., Dalla, S., \& Marriott, D. 2017, MNRAS, 470, 3149

Laitinen, T., Dalla, S., \& Marsh, M. S. 2013, ApJL, 773, L29

Lemen, J. R., Title, A. M., Akin, D. J., et al. 2012, SoPh, 275, 17

Li, Y., Xue, J. C., Ding, M. D., et al. 2018, ApJL, 853, L15

Lin, J., Raymond, J. C., \& van Ballegooijen, A. A. 2004, ApJ, 602, 422

Lin, R. P. 2005, AdSpR, 35, 1857

Lin, R. P., Dennis, B. R., Hurford, G. J., et al. 2002, SoPh, 210, 3

Liu, W., Jin, M., Downs, C., et al. 2018, ApJL, 864, L24

Long, D. M., Harra, L. K., Matthews, S. A., et al. 2018, ApJ, 855, 74

Luhmann, J. G., Mays, M. L., Li, Y., et al. 2018, SpWea, 16, 557

Matthaeus, W. H., Qin, G., Bieber, J. W., \& Zank, G. P. 2003, ApJL, 590, L53

Matthiä, D., Meier, M. M., \& Berger, T. 2018, SpWea, 16, 977

McComas, D. J. 1998, SSRv, 86, 563

McCracken, K. G., Moraal, H., \& Stoker, P. H. 2008, JGRA, 113, A12101

Miroshnichenko, L. I., Pérez-Peraza, J., Vashenyuk, E. V., et al. 1995, Proc. ICRC (Rome,Italy), 4, 34

Mishev, A., \& Usoskin, I. 2016, SoPh, 291, 1225

Mishev, A., Usoskin, I., Raukonen, O., et al. 2018, SoPh, 293, 136

Morosan, D. E., Carley, E. P., Hayes, L. A., et al. 2019, NatAs, 3, 452 
Müller-Mellin, R., Kunow, H., \& Fleißner, V. 1995, SoPh, 162, 483

Murphy, R. J., Dermer, C. D., \& Ramaty, R. 1987, ApJS, 63, 721

Murphy, R. J., \& Ramaty, R. 1984, AdSpR, 4, 127

Omodei, N., Pesce-Rollins, M., Longo, F., Allafort, A., \& Krucker, S. 2018, ApJL, 865, L7

Pick, M., Démoulin, P., Krucker, S., Malandraki, O., \& Maia, D. 2005, ApJ, 625, 1019

Poluianov, S., Usoskin, I., Mishev, A., Shea, M., \& Smart, D. 2017, SoPh, 292, 176

Ramaty, R., Mandzhavidze, N., Kozlovsky, B., \& Skibo, J. G. 1993, AdSpR, 13,275

Reames, D. V. 1995a, AdSpR, 15, 41

Reames, D. V. 1995b, RvGeo, 33, 585

Reames, D. V. 1999, SSRv, 90, 413

Ruffolo, D., Pianpanit, T., Matthaeus, W. H., \& Chuychai, P. 2012, ApJL, 747, L34

Saito, K., Poland, A. I., \& Munro, R. H. 1977, SoPh, 55, 121

Seaton, D., \& Darnel, J. M. 2018, ApJL, 852, L9
Shalchi, A. 2010, ApJL, 720, L127

Share, G. H., Murphy, R. J., White, S. M., et al. 2018, ApJ, 869, 182

Shea, M. A., \& Smart, D. F. 2012, SSRv, 171, 161

Smith, C. W., L'Heureux, J., Ness, N. F., et al. 1998, SSRv, 86, 613

Sokolov, I. V., van der Holst, B., Oran, R., et al. 2013, ApJ, 764, 23

Toptygin, I. N. 1985, Cosmic Rays in Interplanetary Magnetic Fields (Dordrecht: Reidel), 119

Torsti, J., Kocharov, L., Vainio, R., et al. 1996, SoPh, 166, 135

Torsti, J., Riihonen, E., \& Kocharov, L. 2004, ApJL, 600, L83

Torsti, J., Valtonen, E., Lumme, M., et al. 1995, SoPh, 162, 505

Tylka, A. J., Dietrich, W., \& Atwell, W. 2010, in Proc. 38th COSPAR Sci. Assembly (Bremen, Germany), 4

van der Holst, B., Sokolov, I. V., Meng, X., et al. 2014, ApJ, 782, 81

van Haarlem, M. P., Wise, M. W., Gunst, A. W., et al. 2013, A\&A, 556, A2

Veronig, A. M., Podladchikova, T., Dissauer, K., et al. 2018, ApJ, 868, 107

Warren, H. P., Brooks, D. H., Ugarte-Urra, I., et al. 2018, ApJ, 854, 122

Webb, D. F., \& Vourlidas, A. 2016, SoPh, 291, 3725

Yan, X. L., Yang, L. H., Xue, Z. K., et al. 2018, ApJL, 853, L18 